level of CSF, accompanying symptoms, clinical management and prognosis.

Results All patients were HIV negative. The total incidence of JHR in CNS was 7.14\% (41/574, 95\% CI: 5.23-9.65\%), being the most frequent among patients with general paresis. The mean timing of JHR after the initial dose of benzylpenicillin was to start at 6 hours (range: $0.5-13$ ), peak at 8 hours (range: 0.5-20), and subside by 17 hours (range: 10-30). Besides fever and chills, the main symptoms were hallucination, paranoia, aggressive behavior, mental depression, cognitive impairment, confusion, urinary incontinence, stupor, convulsion and seizures in descending order. The JHR was significantly related to higher CSF-VDRL titer, pleocytosis, no usage of antibiotics in the last 6 months $(p<0.05)$. The therapy was stopped with a resolution of seizures in two patients. However, benzylpenicillin was reinstituted uneventfully 3 days later.

Conclusion Higher CSF-VDRL titer, pleocytosis and no recent usage of antibiotics were associated with an increased risk for JHR in CNS. Therapy of neurosyphilis can be continued with intensive surveillance.

Disclosure No significant relationships.

\section{P771 CLINICAL TRIAL OF CEFIXIME FOR THE TREATMENT OF EARLY SYPHILIS - PRELIMINARY RESULTS}

${ }^{1}$ Chrysovalantis Stafylis*, ${ }^{2}$ David Tellalian, ${ }^{3}$ Aishwarya Raich, ${ }^{4}$ Huan Dong, ${ }^{2}$ Pamela Burian, ${ }^{2}$ Cliff Okada, ${ }^{2}$ Carl Millner, ${ }^{2}$ James Carroll, ${ }^{5}$ Cristopher Mejia, ${ }^{5}$ Arberletia Joseph, ${ }^{1}$ Jeffrey Klausner. 'UCLA David Geffen School of Medicine, Infectious Disease, Los Angeles, USA; ${ }^{2}$ AIDS Healthcare Foundation, Department of Medicine, Los Angeles, USA; ${ }^{3}$ UCLA David Geffen School of Medicine, Infectious Diseases, Los Angeles, USA; ${ }^{4}$ Charles R. Drew University of Medicine and Science, LOS Angeles, USA; ${ }^{5}$ AIDS Healthcare Foundation, Public Health Division, Los Angeles, USA

\subsection{6/sextrans-2019-sti.829}

Background Increasing incidence of syphilis in the United States and penicillin shortages internationally call for research on alternative treatment options. In this randomized, multisite, open-label, non-comparative clinical trial, we are evaluating the efficacy of cefixime as treatment of early syphilis.

Methods Eligible participants are 18 years or older, have laboratory confirmed early syphilis (new Rapid Plasma Reagin [RPR] titer $\geq 1: 8$ or 4 -fold titer rise in past 12 months), and no concomitant antibiotic use. Patients with HIV infection must have undetectable viral load in the past 12 months and CD4+ count $\geq 350$ cells $/ \mu$ l. Participants were randomized to receive either $2.4 \mathrm{M}$ IU benzathine penicillin $G$ intramuscularly once or cefixime $400 \mathrm{mg}$ orally twice a day, for ten days. Participants return for follow-up at 3, 6, and 12 months posttreatment for laboratory testing. The main outcome is a 4fold RPR titer decrease at 6 months post-treatment.

Results To date, 27 participants (15 penicillin, 12 cefixime) are enrolled. The majority of the study population is men (26/27), Latino (15/27), and HIV-infected (25/27). Eight participants completed their 3-month follow up (4 cefixime/4 penicillin). In the cefixime arm, $3 / 4$ participants had an equal or greater than four-fold decrease in the RPR titer, and $1 / 4$ had a two-fold decrease. In the penicillin arm, 2/4 participants had an equal or greater than 4 -fold decrease in the RPR titer, $1 / 4$ had a two-fold decrease, and $1 / 4$ is missing data.
Conclusion Enrollment is still open and data collection ongoing. Initial results are encouraging.

Disclosure No significant relationships.

\section{P772 GASTRIC SYPHILIS: A CASE OF GASTRIC SYPHILIS DEVELOPED INTO NEUROSYPHILIS}

${ }^{1}$ Lin Zhu*, 'Mei Shi, ${ }^{2}$ Rui-Rui Peng, ${ }^{2}$ Xin Gu, ${ }^{2}$ Zhi-Fang Guan, ${ }^{2}$ Pingyu Zhou. ${ }^{1}$ Shanghai Skin Disease Hospital, Shanghai Skin Disease Hospital, Shanghai, China; ${ }^{2}$ Sexually Transmitted Disease Institute, Shanghai Skin Disease Hospital, Shanghai, China

\subsection{6/sextrans-2019-sti.830}

Background Syphilis caused by the spirochetes Treponema pallidum, which transmitted mainly through sexual contact and blood transmission. The spirochetes spread from the damaged skin to bloodstream, at which point any organ may be affected included bone, central nervous system, and visceral organs-the stomach. Therefore, when treating patients with syphilis, the clinician must rule out the involvement of other organs. Gastric syphilis is a rare presentation of infection with Treponema pallidum, which has no-specific symptoms. Gastric syphilis may occur in any phase of syphilis, predominantly in the secondary $(50 \%)$, but rarely in the third(6\%). There are no standard recommendations specifically address the treatment of gastric syphilis. The general approach is to treat according to the stage of the disease. However, if the clinician does not have experience the disease will be misdiagnosed. Here, we will describe a case of a patient with gastric involvement, whose symptom repeated after conventional treatment.

Methods The patent's history, clinical examination, biology, cerebrospinal fluid,blood nest PCR findings and reatment are reported. We also discuss the profit treatment options.

Results we described a case of a patient with the complaint of epigastralgia, pyrosis, and regurgitation. The gastroscopy revealed multiple ulcerative lesions at the antrum, gastric angle and duodenal ampulla, in which suspected cancer. Mucosal biopsy revealed an inflammatory infiltrate with multiple mature plasma cells. Immunohistochemistry and a nested PCR showed the presence of Treponema pallidum in the gastric tissues. But, with conventional treatment, the symptom repeated. Conclusion The patient was diagnosed as having neurosyphilis with gastric syphilis.Her epigastric pain improved on the seventh day of the treatment. Follow-up gastroscopic findings tree months after diagnosis showed improved ulcerative lesions on gastric antrum and duodenal ampulla. The CSF examination was negative.

Disclosure No significant relationships.

\section{P773 INCREASED DETECTION RATES OF PRIMARY SYPHILIS BY PCR IN A PROVINCIAL LABORATORY}

Prenilla Naidu*, Fiona Ko. Provincial Laboratory for Public Health, Edmonton, Canada

\subsection{6/sextrans-2019-sti.831}

Background North America is experiencing an exponential rise in the numbers of infectious syphilis cases. The United States of America had 9.5/100,000 cases in 2017 which is a 10.5\% increase from 2016 and a $72.7 \%$ increase from 2013. This trend is no different in Canada with Alberta being particularly 